ISSN: $2394-2258$

Available at http://scientificadvances.co.in

DOI: http://dx.doi.org/10.18642/ijamml_7100122153

\title{
OPEN PROBLEMS ON INTEGRAL SUM LABELLINGS
}

\section{Tian-Rui Ye and Haiying Wang}

Beijing No. 4 High School International Campus, Beijing, 100031, P. R. China

School of Science, China University of Geosciences (Beijing), Beijing, 100083, P. R. China

\begin{abstract}
Let $G=(V, E)$ be a simple graph and $Z$ be the set of all integers. An integral sum graph of a set $S$ of integers as the graph $G^{+}(S)$ having $S$ as its vertex set, with two vertices adjacent whenever their sum is in $S$. A graph $G^{+}(S)$ so obtained is called an integral sum graph. In other words, an integral sum graph $G^{+}(S)$ of a finite subset $S \subset Z$ is the graph $(S, E)$ with $u v \in E$ if and only if $u+v \in S$. And $S$ is called an integral sum labelling of $G^{+}(S)$. In the paper, many obtained conclusions are summarized and relevant open problem is raised.
\end{abstract}

${ }^{*}$ Corresponding author.

E-mail address: whycht@126.com (Haiying Wang).

Copyright (c) 2020 Scientific Advances Publishers

2020 Mathematics Subject Classification: 05C78.

Submitted by Haitao Xu.

Received August 15, 2020

This work is licensed under the Creative Commons Attribution International License (CC BY 3.0).

http://creativecommons.org/licenses/by/3.0/deed.en_US

Open Access (C) (P) 
Keywords: sum graph, integral sum graph, integral sum labelling.

\section{Introduction}

Let $G=(V, E)$ be a simple graph with the vertex set $V$ and edge set $E$. Let $Z$ and $N$ denote the set of all integers and the set of all positive integers, respectively. The concepts of the integral sum graph and the sum graph were introduced by Harary ([2], [3]). The related research is mainly applied in data storage and algorithm acceleration of computer.

An integral sum graph of a set $S$ of integers as the graph $G^{+}(S)$ having $S$ as its vertex set, with two vertices adjacent whenever their sum is in $S$. A graph $G^{+}(S)$ so obtained is called an integral sum graph. In other words, an integral sum graph $G^{+}(S)$ of a finite subset $S \subset Z$ is the graph $(S, E)$ with $u v \in E$ if and only if $u+v \in S$. And the set $S$ is called an integral sum labelling of $G^{+}(S)$. If we replace $Z$ with $N$, the notions of the sum graph, the sum labelling and the sum number are got respectively.

To simplify the notations, throughout this paper we may assume that the vertices of $G$ are identified with their labels. All other notations and terminologies are referred to [1].

In this paper, we mainly talk about the integral sum labelling of a graph. 


\section{Main Results}

Let $P_{n}$ and $C_{n}$ denote Path and Cycle with $n$ vertices, respectively.

Example 1. $P_{4}$ is one integral sum graph (see Figure 1).

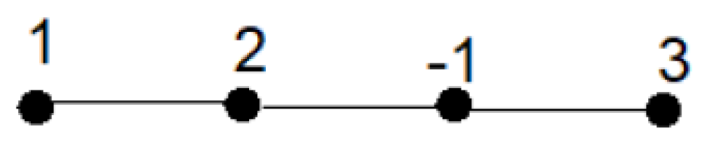

Figure 1. $P_{4}$.

Example 2. $C_{5}$ is one integral sum graph (see Figure 2).

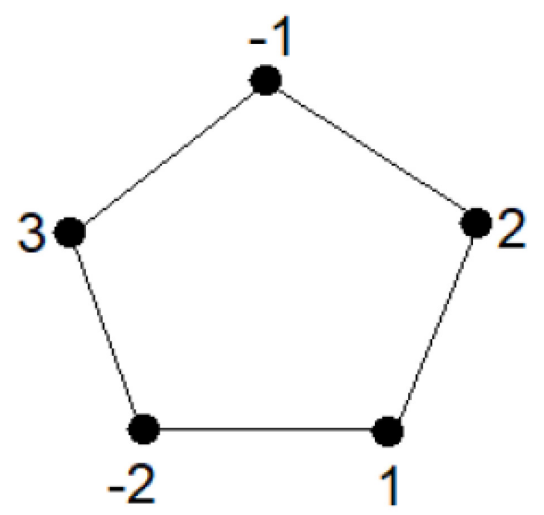

Figure 2. $C_{5}$.

According to the definitions above, $\{1,2,-1,3\}$ and $\{3,-2,1,2,-1\}$ are the corresponding integral sum labels of $P_{4}$ and $C_{5}$. Thus, two graphs in Figures 1 and 2 are the integral sum graphs.

As we know, it is difficult to determine whether a graph is an integral sum graph. Even a graph is known to be an integral sum graph, its topological properties are very important and it is hard to obtain for us. These questions are still open. 
Problem 1. How to determine if a graph is an integral sum graph.

Problem 2. Characterize the integral sum graph.

\section{Acknowledgement}

The research is supported by NSFC (No. 11701530).

\section{References}

[1] J. A. Bondy and U. S. R. Murty, Graph Theory with Applications, MacMillan, New York, NY, 1976.

[2] F. Harary, Sum graph and difference graphs, Congressus Numerantium 72 (1990), 101-108.

[3] F. Harary, Sum graph over all the integers, Discrete Mathematics 124(1-3) (1994), 99-105.

DOI: https://doi.org/10.1016/0012-365X(92)00054-U 